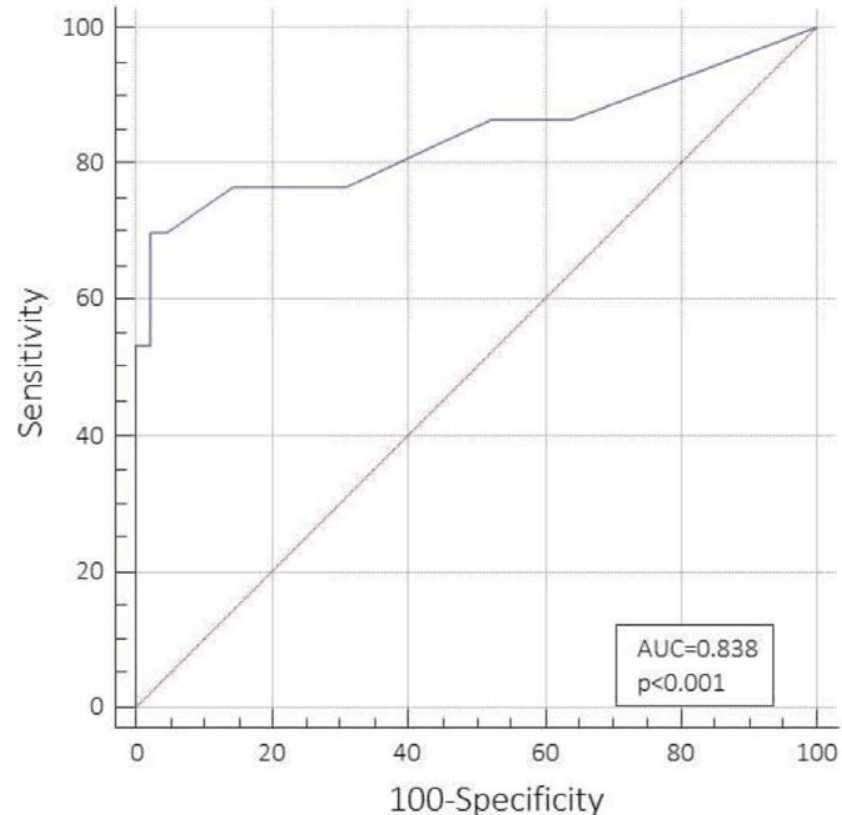

Figure 2. Area under the ROC curve to determine the number of B-lines at LUS to define a significant RA-ILD, applying the $10 \%$ of fibrosis at chest HRCT measured by OsiriX as external criterion.

Conclusion: The present study provided data to determine the number of B-lines to identify a significant RA-ILD. LUS may represent a useful technique to select RA patients to be assessed by chest HRCT.

References:

[1] Tardella $\mathrm{M}$, et al. Ultrasound $\mathrm{B}$-lines in the evaluation of interstitial lung disease in patients with systemic sclerosis: Cut-off point definition for the presence of significant pulmonary fibrosis. Medicine (Baltimore). 2018;97(18):e0566.

[2] Salaffi $F$, et al. High-resolution computed tomography of the lung in patients with rheumatoid arthritis: Prevalence of interstitial lung disease involvement and determinants of abnormalities. Medicine (Baltimore). 2019;98(38):e17088.

Disclosure of Interests: Marco Di Carlo: None declared, Marika Tardella: None declared, Emilio Filippucci Speakers bureau: Dr. Filippucci reports personal fees from AbbVie, personal fees from Bristol-Myers Squibb, personal fees from Celgene, personal fees from Roche, personal fees from Union Chimique Belge Pharma, personal fees from Pfizer, outside the submitted work., Fausto Salaffi: None declared DOI: 10.1136/annrheumdis-2020-eular.4214

\section{SAT0071 SUBCLINICAL SYNOVITIS IN ARTHRALGIA: HOW OFTEN DOES IT RESULT IN CLINICAL ARTHRITIS? A LONGITUDINAL STUDY TO REFLECT ON STARTING POINTS FOR DMARD TREATMENT}

C. Rogier ${ }^{1}$, F. Wouters ${ }^{2}$, L. Van Boheemen ${ }^{3}$, D. Van Schaardenburg ${ }^{3}$, P. De Jong ${ }^{1}$, A. Van der Helm-van Mil ${ }^{1,2} .{ }^{1}$ Erasmus MC, Rheumatology, Rotterdam, Netherlands: ${ }^{2}$ Leiden University Medical Centre (LUMC), Rheumatology, Leiden, Netherlands; ${ }^{3}$ Reade, Rheumatology, Amsterdam, Netherlands

Background: Clinically apparent arthritis is mandatory for diagnosing and classifying RA. It is often used as endpoint in arthralgia cohorts and as a starting point for DMARD therapy in clinical practice. In recent literature subclinical synovitis, visualized with MRI or ultrasound, is increasingly used as a starting point for DMARD therapy in absence of clinically apparent arthritis. However, not all patients with a subclinical synovitis will develop clinically apparent arthritis, and thus may be overtreated. It has even been suggested to replace the entry-criterion of clinical arthritis by subclinical synovitis within the 2010 classification criteria for RA to diminish overtreatment. However this might lead to an overclassification of the disease. Because of aforementioned reasoning we aimed to evaluate the risk of overtreatment of these approaches and therefore performed a longitudinal study in three observational arthralgia cohorts.

Objectives: To determine the frequency of non-progression to clinical arthritis in patients with subclinical synovitis, also after considering the 2010-criteria.

Methods: Three individual cohorts of arthralgia patients without clinically apparent arthritis ( $n=166,473$ and 168) were followed for 1-year on the development of inflammatory arthritis (IA). At baseline subclinical synovitis in hands or feet was visualized with ultrasound (US) (defined as greyscale $\geq 2$ and/or power-doppler $\geq 1$ ) in cohort 1 and 3 and MRI (synovitis score $\geq 1$ by two readers) in cohort 2 . For all patients with subclinical synovitis the proportion of progressors ('true positives') and non-progressors ('false positives') were determined. The same analysis was done in the subgroup of patients that fulfilled the 2010 criteria for RA, if subclinical synovitis was used as entry criterion. Analyses were stratified for ACPA.

Results: At baseline $36 \%, 41 \%$ and $31 \%$ of patients had subclinical synovitis. Of the ACPA-positive arthralgia patients with subclinical synovitis $46 \%, 56 \%$ and $29 \%$ respectively developed IA, whereas $54 \%, 44 \%$ and $71 \%$ did not progress. Within ACPA-negative arthralgia patients with subclinical synovitis $34 \%, 15 \%$ and $10 \%$ developed IA; whereas $66 \%, 85 \%$ and $90 \%$ did not progress (Figure $1 \mathrm{~A}$ ). Similar results were seen in the subgroup of patients that fulfilled the 2010 criteria with subclinical synovitis as entry criterion (Figure 1B).

\section{A}

Patients presenting with arthralgia and imaging detected subclinical synovitis
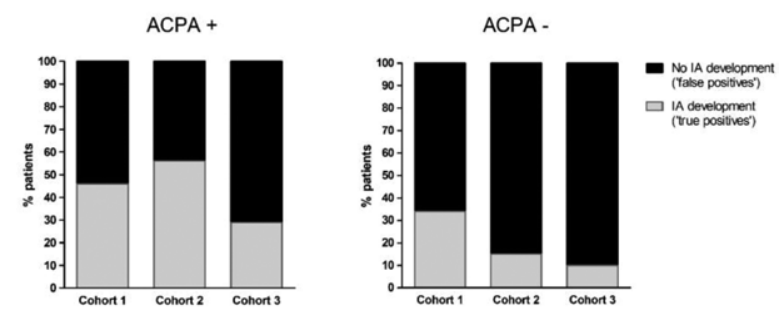

B

Patients presenting with arthralgia, imaging detected subclinical synovitis and $\geq 6$ points on the 2010-criteria (with subclincal synovitis as entry-criterion)
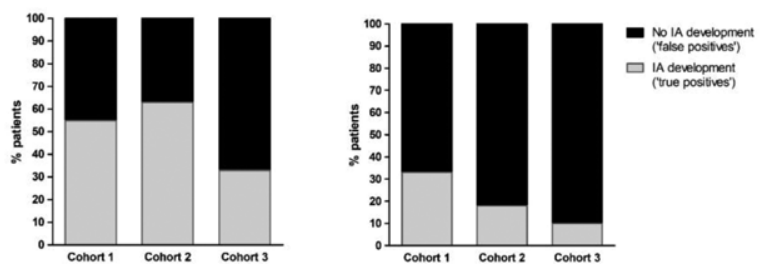

Figure 1. Percentage of arthralgia patients with subclinical synovitis that did and dic not develop IA, in three independent cohorts: (A) Percentage of patients with subclinical synovitis that did and did not progress to IA after one-year follow-up, stratified for ACPA status. (B) Percentage of patients with subclinical synovitis and $\geq 6$ points at baseline that did and did not progress to IA after one-year follow-up stratified for ACPA status.

Conclusion: Replacing clinical arthritis by subclinical synovitis in arthralgia introduces a high false-positive rate: $44-71 \%$ (ACPA-pos) and 66-90\% (ACPAneg) of patients with subclinical synovitis did not develop clinically apparent arthritis within one year. Applying the 2010-criteria in this setting did not diminish the false positive rate. Starting DMARDs in patients without clinical synovitis may therefore introduce considerable overtreatment.

Acknowledgments *: C Rogier and F Wouters contributed equal to this study Disclosure of Interests: None declared DOI: 10.1136/annrheumdis-2020-eular.3157

\section{SAT0072 THE IMPACT OF COMORBIDITIES ON ABSENTEEISM, PRESENTEEISM AND EMPLOYMENT STATUS IN PEOPLE LIVING WITH RHEUMATOID ARTHRITIS}

A. Bradshaw ${ }^{1}$, A. Bosworth ${ }^{2}$, K. Walker-Bone ${ }^{3}$, L. Lunt ${ }^{1}$, S. Verstappen ${ }^{1}$.

${ }^{1}$ Centre for Musculoskeleta Research, Manchester, United Kingdom;

${ }^{2}$ NRAS, Maidenhead, United Kingdom; ${ }^{3} \mathrm{MRC}$ Verssus Arthritis Centre for Musculoskeletal Health and Work, Southampton, United Kingdom

Background: Many people with rheumatoid arthritis (RA) have comorbidities. However, there is limited research on the impact of multimorbidity on absenteeism (e.g. sick leave) and presenteeism (i.e. reduced productivity while at work due to ill health) in people with RA.

Objectives: i) to explore the impact of comorbidities on absenteeism and presenteeism in patients with RA and ii) to evaluate the association between multimorbidity and employment status.

Methods: A cross-sectional survey was conducted by the National Rheumatoid Arthritis Society (NRAS), UK, collecting information on: demographics, education, employment status (i.e. employed (Empl), stopped/retired early because of RA (Stop_RA), stopped/retired early because of other health issues (Stop_Health)), and disease related variables (e.g. symptom duration, rheumatoid arthritis impact of disease (RAID) questionnaire). Participants were asked to report whether they 
had or were treated for any of 15 predefined comorbidities (categorised into 0, 1, 2, 3 , or $\geq 4$ (Table)). Percentage of number of hours missed due RA (i.e. absenteeism) and presenteeism (10-point Likert scale) were assessed using the Work Productivity and Activity Impairment Questionnaire (WPAI-RA). For the purpose of this study both absenteeism and presenteeism outcomes were dichotomized (no presenteeism/absenteeism versus any) and only patients aged $<65$ yrs were included. Logistic regression analysis were applied to assess the association between number of comorbidities and absenteeism/presenteeism, adjusting for the categorical variables age, gender and education. Chi2-square test was applied to assess frequencies of individual comorbidities between the three employment status groups. Results: 868 participants were included; $91.7 \%$ women with a median symptom duration of 8.3 years [IQR 4.4-13.7]. The average RAID score was 5.2 (SD 2.2). $80.4 \%$ were in paid employment, including those currently on sick leave, $16.9 \%$ stopped early because of their RA and $2.7 \%$ reported stopping early because of other health reasons. In those employed most commonly occurring comorbidities were: back pain $(28.8 \% \%)$, osteoarthritis $(21.5 \%)$, depression $(26.3 \%)$ and anxiety $(22.6 \%)$. Compared to people with RA with no comorbidities, the odds associated with time off work due to RA increased from 1.7 up to 3.4 with increasing number of comorbidities (Table). Although a similar trend was observed for presenteeism, the effect sizes were smaller. Significant differences $(p<0.05)$ in frequencies of the following comorbidities were observed between the three employment status groups (Empl, Stop_RA, Stop_Health, respectively): heart disease $(3.9 \%, 7.9 \%, 20.0 \%)$, blood pressure $(18.0 \%, 29.5 \%, 36.7 \%)$, lung disease $(5.7 \%, 16.3 \%, 26.7 \%)$, diabetes $(4.4 \%, 4.2 \%, 26.7 \%)$, ulcer $(6.1 \%, 11.1 \%$, $13.3 \%)$, cancer $(3.3 \%, 2.6 \%, 13.3 \%)$, depression $(26.3 \%, 33.6 \%, 50.0 \%)$, OA $(21.5 \%, 44.7 \%, 63.33 \%)$, and back pain $(28.8 \%, 48.4 \%, 60.0 \%)$.

\begin{tabular}{|c|c|c|c|c|c|}
\hline \multirow[b]{2}{*}{ Number of comorbidities } & \multirow[b]{2}{*}{$\mathbf{N}$} & \multicolumn{2}{|c|}{ Absenteeism (yes/no) } & \multicolumn{2}{|c|}{ Presenteeism (yes/no) } \\
\hline & & OR & $95 \% \mathrm{Cl}$ & OR & $95 \% \mathrm{Cl}$ \\
\hline 0 & 206 & Ref. & & Ref. & \\
\hline 1 & 174 & 1.70 & $\begin{array}{l}1.06- \\
2.71\end{array}$ & 1.66 & $1.00-2.73$ \\
\hline 2 & 136 & 1.77 & $\begin{array}{l}1.08- \\
2.92\end{array}$ & 1.99 & $1.13-2.86$ \\
\hline 3 & 85 & 1.75 & $\begin{array}{l}1.00- \\
3.08\end{array}$ & 1.53 & $0.82-2.86$ \\
\hline 4-15max & 86 & 3.38 & $\begin{array}{l}1.98- \\
5.78\end{array}$ & 2.64 & $1.28-5.44$ \\
\hline
\end{tabular}

OR=odds ratio; $95 \% \mathrm{Cl}=95 \%$ confidence interval; Comorbidities included: heart disease, blood pressure, lung disease, diabetes, ulcer or stomach disease, kidney disease, liver disease, anaemia or other blood disease, cancer, depression, anxiety, OA, back pain, osteoporosis and Sjögren. Bold figures $P<0.05$

Conclusion: Although the study is cross-sectional and no temporal association can be determined, this study shows that not only personal and work related contextual factors should be considered when preventing worker productivity loss, but also other comorbidities.

Disclosure of Interests: A. Bradshaw: None declared, Ailsa Bosworth Speakers bureau: a number of pharmaceutical companies for reasons of inhouse training, advisory boards etc., K. Walker-Bone: None declared, Laura Lunt: None declared, Suzanne Verstappen Grant/research support from: BMS, Consultant of: Celltrion, Speakers bureau: Pfizer

DOI: 10.1136/annrheumdis-2020-eular.5467

\section{SAT0073 MYOPENIA AGGRAVATES RHEUMATOID ARTHRITIS IN ELDERLY PATIENTS AS A MEDIATOR}

J. D. MA ${ }^{1}$, C. Chen ${ }^{1}$, J. Z. Lin ${ }^{1}$, Q. H. LI ${ }^{1}$, L. F. Chen ${ }^{1}$, Y. H. Xu' ${ }^{1}$, D. H. Zheng ${ }^{1}$, L. Dai ${ }^{1}$. ${ }^{1}$ Sun Yat-Sen Memorial Hospital, Sun Yat-Sen University, Rheumatology, Guangzhou, China

Background: Ageing affects different systems resulting in a special clinical phenotype of rheumatoid arthritis (RA) in elderly patients who are characterized by higher level of systemic inflammatory and poor function. It also leads to loss of muscle mass causing functional limitation and reduced quality of life. However, little is known about muscle loss in growing elderly RA patients.

Objectives: To explore the characteristics of muscle mass and clinical significance in elderly RA patients.

Methods: Consecutive RA patients were recruited and clinical data including disease activity (DAS28-CRP), function (HAQ-DI) and radiographic indicators (modified Sharp score) were collected. The mass and distribution of muscle were assessed by bioelectric impedance analysis. Myopenia was defined as appendicular skeletal muscle mass index (ASMI) $\leq 7.0 \mathrm{~kg} / \mathrm{m}^{2}$ in men and $\leq 5.7 \mathrm{~kg} / \mathrm{m}^{2}$ in women.
Results: (1) There were 643 RA patients recruited with $82.3 \%$ female, mean age $49.7 \pm 12.9$ years and median disease duration 48 (IQR 21,108) months. There were $414(64.4 \%)$ RA patients with active disease (DAS28-CRP $\geq 3.2$ ) and 229 (35.6\%) with remission. (2) There were 165 (25.7\%) elderly RA patients (age $\geq 60$ years) with mean age $65.1 \pm 4.5$ years. Compared with young patients (age $<60$ years), elderly RA patients had significantly higher disease activity indicators including PtGA, PrGA, ESR, CRP, DAS28-CRP, DAS28-ESR, SDAl and $C D A I$, higher $H A Q-D I(0.38$ vs. 0.13$)$ and higher modified total Sharp score (mTSS, 16 vs. 9, all $P<0.001)$. There were $288(44.8 \%)$ RA patients with myopenia and elderly RA patients had higher proportion of myopenia than young patients (54.5\% vs. $41.4 \%, P=0.003)$. (3) Among 4 subgroups according to age and ASMI, elderly RA patients with myopenia ( $n=90,14.0 \%)$ had significant higher DAS28-CRP (3.6 vs. 3.0), higher HAQ-DI (0.50 vs. 0.12 ) and higher mTSS ( 21 vs. 7 ) than those in young patients without myopenia ( $n=280$ $43.5 \%$ ), and had higher mTSS (21 vs. 10) than those in elderly patients without myopenia ( $n=75,11.7 \%$, all $P<0.0083)$. (4) Adjusted for confounding factors including gender, disease duration, BMI, smoking habit, RF, ACPA and treatment naïve, multiple linear regression analysis showed that age was positively correlated with DAS28-CRP $(\beta=0.010), \mathrm{HAQ}-\mathrm{DI}(\beta=0.003)$ and $\mathrm{mTSS}(\beta=0.005$, all $P<0.05)$, while ASMI was negatively correlated with DAS28-CRP $(\beta=-0.445)$ HAQ-DI $(\beta=-0.124)$ and mTSS $(\beta=-0.247$, all $P<0.001)$. (5) Mediation analysis showed that old age ( $\geq 60$ years) had total effect on DAS28-CRP $(\beta=0.353)$ HAQ-DI $(\beta=0.132)$ or mTSS $(\beta=7.909$, all $P<0.05)$, but no direct effect on them (all $P>0.05$ ). ASMI fully mediated the associations between old age and DAS28CRP, HAQ-DI or mTSS.

Conclusion: Half of elderly RA patients manifest myopenia which aggravates the whole disease of disease activity, joint function and destruction as a mediator. Myopenia, a neglected comorbidity in elderly RA should be emphasized.

Funding: This work was supported by National Natural Science Foundation of China (81801606 and 81971527,), Natural Science Foundation of Guangdong Province (2018A030313541 and 2019A1515011928), Science and Technology Program of Guangzhou (201904010088).

Disclosure of Interests : None declared

DOI: 10.1136/annrheumdis-2020-eular.1390

\section{SAT0074 RHEUMATOID ARTHRITIS AT TREATMENT WITH BDMARD OR TSDMARD: VACCINATION RATES AND INCIDENCE OF RESPIRATORY INFECTIOUS DISEASES, RESULTS FROM A COHORT}

R. Dos Santos Sobrín ${ }^{1}$, E. Perez-Pampín ${ }^{1}$, N. Pérez Gómez ${ }^{1}$, A. Mera Varela ${ }^{1}$ ${ }^{1}$ Clinical University Hospital in Santiago de Compostela, Rheumatology Department, Santiago de Compostela, Spain

Background: Vaccination regimes have been evaluated for long time in rheumatic diseases, being a strong recommendation to vaccinate against Influenza and Pneumococcus (13 and 23-valent). Rheumatoid arthritis (RA) patients have higher rates of infectious diseases, caused by many reasons, being patient's comorbidities, rheumatic disease and treatments used the most important ${ }^{1-2}$.

Objectives: To analyze the incidence of respiratory infectious diseases in these patients regarding for vaccination status. Also prove the degree of accomplishment of vaccination calendar.

Methods: Patients diagnosed of RA at treatment with bDMARD or tsDMARD, in Rheumatology Department of aforementioned hospital, during Influenza vaccination campaign in 2018 (October 2018 - February 2019) were included. Clinical, demographic and therapeutic data were reviewed. Stata 15.1 was used to perform statistical analysis.

Results: 237 patients finally fulfilled inclusion criteria, excluding deceases or finished treatment (460 patients were diagnosed of RA and 954 patients conform all bDMARD and tsDMARD of Rheumatology Department). Mean age at beginning of vaccination campaign was 61,5 years old (SD 13,6). $79 \%$ were female. Mean time of diagnosis was 15,4 years (SD 9,4). $79,7 \%$ patients receive Influenza vaccine, although higher rates were found in Pneumococcal vaccine (86,9\% 13-valent and 81,8\% 23-valent). Most patients were at treatment with anti-TNF $(57,2 \%$, the most prevalent was etanercept $27,5 \%$ followed by adalimumab $11,0 \%$ and infliximab 10,2\%). csDMARD concomitant was achieved by $67,4 \%$ patients (methotrexate $73 \%$ ) and $61 \%$ receive corticosteroids. Only 3 patients got hospitalized by pneumonia. As opposed, 39 patients suffer from a respiratory infectious disease without hospitalization (mean of 1,33 infections/ patient). After multivariate analysis, only 13-valent Pneumococcal vaccine is related statistically significant with higher rates of respiratory infectious diseases (Chi2=6,25 p=0,012; OR 2,86 Cl95\% 1,22 to 6,68). Other variables analyzed were kind of bDMARD/tsDMARD, Influenza vaccine, 23-valent Pneumococcal vaccine, concomitant csDMARD/corticosteroids, but no relationship was found. Conclusion: Vaccination status is still incomplete in majority of rheumatic patients. Its benefits have been explained in a variety of studies. That is the 